\title{
Do you speak 'urban design'?: Intermediations between grammar of space and the fragments of city-text
}

\author{
Murat Çetin \\ Department of Architecture, \\ King Fahd University of Petroleum and Minerals, \\ KFUPM, College of Environmental Design, \\ P.O. Box 910, 31261, Dhahran, Saudi Arabia \\ Fax: +966-(0)3-860-32-10 \\ E-mail: murat@kfupm.edu.sa \\ E-mail: mcetin01@gmail.com
}

\begin{abstract}
It is aimed to probe whether underlying formal regularities in urban metamorphosis may constitute potential basis of urban-architectural interventions. Urban design is described as a formal language. Underlying principles of urban transformation are argued as what constitute the grammatical structure of urban form and its change. Thus, socio-cultural significance of grammatical encoding inherent in the morphology of urban space is emphasised whereby geometrical relationships are utilised as instruments of spatial analysis. Urban transformation is analysed in terms of rule-based, compositional systems called 'formal grammars'. It is observed that there is a linguistic logic composed of an initial form of the design, a set of possible rules applied to this initial form, and recursive structures which define the sequence, order and location of the rules applied to it. Formal grammars are proposed as a helpful instrument of understanding to the broader framework of townscape and morphological analyses prior to urban design.
\end{abstract}

Keywords: urban design; morphology; semiology; transformation; formal grammars.

Reference to this paper should be made as follows: Çetin, M. (2011) 'Do you speak 'urban design'?: Intermediations between grammar of space and the fragments of city-text', Int. J. Arab Culture, Management and Sustainable Development, Vol. 2, No. 1, pp.83-100.

Biographical notes: Murat Çetin has completed his undergraduate and graduate studies in Middle East Technical University, Department of Architecture. He was awarded by the Higher Education Council for a scholarship to conduct his doctoral studies at Sheffield University in UK. He participated in various international conferences and published papers in journals and books. After his return, he taught at Balikesir University, Department of Architecture and Yeditepe University as an Assistant Professor. $\mathrm{He}$ also won a citation awards in one urban design competition and one architectural conservation project competition. He currently teaches at King Fahd University of Petroleum and Minerals. 


\section{Introduction}

Whether urban transformation could be a form of 'encoded cultural transmission' has been the departure point of this study towards finding out constant principles on which urban interventions could be based. This main objective might well be accompanied by the following questions: could there be 'constant principles' in the transformation of all phenomena? Could there be parallels between the ruptures in the continuity of 'formal transformation' and those in the 'semiological disintegration' of urban environment? Could 'human programming ability' be reflected on the way man shape and transform their environment as it is reflected on language, pattern recognition and music? Could, therefore, the evolution of a particular location be defined in 'linguistic and grammatical' terms? Common terrain of all these questions is that of underlying morphological regularities within the formal language of change. As the title implies, this article points out a growing awareness of grammatical structure of this language among designers.

Therefore, this paper investigates the grammatical regularities of urban space on the basis of the analogy between language and urban design. Thus, grammatical approach is argued as an alternative theoretical approach to urban renewal which currently seems to have besieged in the boundaries of the 'new versus old' debate. The paper argues whether deeper structures of architectural and urban space may provide a sound theoretical framework, design freedom and a realm of rational debate and creative intuition (Hillier, 1993).

The scope of the current article gains further significance especially when form and content is to be considered separately (Simmel, 1955). It is intended to establish a common ground (for a multi-disciplinary approach) to the issue of 'intervention into existing contexts'. In that sense, plausibly constant recursivities of transformation are investigated as potential basis of such intervention. Thus, urban design as a language of renewal, and formal grammar as its underlying set of principles play a central role in such evaluation of urban transformation for deriving principles of intervention.

One of the main arguments in such alternative attempt is based on the observation that space, hitherto, has not been tackled as an objective entity in itself, but tied in some way to human agency. It is built so inseparably into socio-cultural life that its forms become nearly invisible. One would like to return, at this point, to Geoffrey Scott's argument on the 'external assignment of social, symbolic and experiential meanings on intrinsic qualities of form mainly by literature', and to point out certain common grammatical characteristics among these external values as well as intrinsic qualities. Therefore, it is alternatively suggested to separate form and content of 'urban space' temporarily, enabling to establish parallels between the ruptures in the continuity of 'formal transformation' and those in the 'semiological disintegration' of urban environment.

Since 'urban morphology' can be assumed as a 'language of forms' encoding the transformation of socio-cultural attributes related to the urban space, scholars in urban-architectural sciences should concentrate on deciphering the existing urban morphology, while the urban designers (and architects) should focus on utilising these analyses in order to be able to encipher their formal vocabulary on the basis of these grammatical deep-structures of urban space. These analyses should be based on the recursivities of 'rule based geometry'. The interactions between the geometrical and the semiological components in space should be comperatively scrutinised in a chronological understanding. 


\section{Urban morphology as the reflection of shared and underlying formal values}

Within the morphological framework, the patterns of urban form, which could be assumed as the manifestation of its composite character, reflect multiple decisions by its inhabitants and participants of its planning, as well as its activities. Be that as it may, one can not fail to ask if urban form could be conceived as another form of encoded cultural transmission. Thereby, patterns, as the underlying formal regularities, may encipher some sort of information about urban-architectural traditions as most of the patterns in many arts and crafts reveal clues about social factors behind cultural traditions. What is beyond doubt is that underlying formal regularities appears to hinge on shared values of urban design between global and local cultures.

Readers' attention should also be drawn to the notion of 'preservation' in urban environment as another agent in transmission of culture and tradition. The current overload of frozen practice of preservation should not obscure the fact that conservation of the 'principles of creating' serves as a strong foundation for dissolving the opposite registers of preservation and renewal within a unified form. Because, 'forms' might change but 'relations between the rules' (i.e., principles) that are applied to forms seem to remain constant.

\section{Excavation of recurring syntax through layers of urban metamorphosis}

The study attempts, here, to explore formal attributes of city in linguistic terms and the continuity of its urban-architectural traditions in terms of the recursivity of the patterns that constitute urban context, space, and townscape traditions. Indeed, the boundary of design knowledge is one of form and its generating structures both essential to making of architecture. Also, many examples throughout the human history prove that form may transcend external influences and may have a nearly independent nature of its own to which those external factors can be attributed. Referring to Rykwert (1976), we can further derive that there are timeless qualities and the semi-autonomous shared architectural language of form (Guadet, 1894; Gosling and Maitland, 1984; Thiis-Evansen, 1988). Also, former research (e.g., Doxiadis, 1972; Herbert and Sanders, 1994; Wightman, 1997; Adams, 1999) has so far intriguingly proved form and geometry essential in revealing underlying - and sometimes implicit - design principles with regard to its social, symbolic and experiential contexts. Therefore, one must not underestimate the potentials of formal analysis because reading architecture is a prerequisite to composing it. In fact, geometry is, thus, an essential tool in both reading and composing the language of urban design as a reflection of culture.

Chomsky's view that language is an instinct with a logic genetically and universally pre-programmed into human brain shows the high possibility of its impact on the way we design our environment. Whether it is genetic or adopted, programming skills seem to be at the very heart of human linguistic ability. On the basis of architecture - language analogy, it is of interest to examine whether urban renewal can be defined in linguistic terms, and whether a seemingly complex phenomenon, such as 'urban context in time', could be defined in terms of grammar. Hitherto, Saussureian understanding has claimed to be capable of explaining diverse aspects of culture, including architecture, in terms of 
language. Simmelian thinking may further enrich and clarify inherent ambiguities of the structuralist stance by redefining its boundaries within the domain of the form of cultural phenomena, particularly urban form.

Along another avenue, cyclic nature of city growth, in terms of its activities (Whitehand, 1987), suggest pattern-like characteristics. Rykwert (1976) also explains this common nature by suggesting that 'human markings known are bare expression of rhythmic values'. Thus, formal and diverse non-formal aspects of urban form and its transformation seem to be linked on the basis of these patterns and their repetitive nature. One may also devise ways of describing 'tradition' as pattern problem under the light of discussions above. Rule-based systems of design and pattern-like reading of the fragments of city seem to call for some sort of correlation. As science searches for patterns in all phenomena, it is intended to describe a complex and irregular phenomena like urban transformation in terms of such regularities.

When context and tradition are defined as pattern problems, 'meaning' may become a function or a code. Thereby, codes and connotative subcodes, which make different readings possible, are engaged in a communicative function. Hence, what is communicated through these formal codes, at different levels of codification, suggests a translation of this formal-code language. Then, one is confronted with the necessity for semantic interpretation for syntactic study (Eco, 1997) of urban form. Syntactic dissolution of the morphological aspect of city has to be related to its multiplicity of meanings in different contexts. The meaning content of urban environment can be searched within the domain of city understood as remnants of lost orders and fragments of never realised totalities. Thus, urban meaning seems to lie within broken symmetries thereby city becomes a fragmented text. Therefore, inflections of a 'hypothetically undisturbed order', in formal grammar analyses, seem to be related to 'hidden meanings encoded in the fragments of the city-text'. Architectural science may intend to pursue such connections through linguistic methodology based on Conzenian urban-architectural morphology.

\section{Science of urban design as the language of urban renewal}

When urban design is conceived as a scientific discipline, it can be expected not only to investigate the underlying tectonics through the methodological analysis of processes, regularities or recursivities within the urban-architectural appearances, but also to give any plausible underlying pattern a meaning by sound references to socio-cultural studies. Thus, scientific study of urban design requires a re-reading of 'existing fabric' and 'academic knowledge' that is hitherto built via a speculative understanding.

Friedman (1975), in his search for a 'scientific architecture', defines city as an organism whose mechanism bears a certain code of information that is composed of a network and a set of rules. He emphasises regularities as the structural basis of urban mechanism. The principles of codification, speculation, simulation and interpretation, inherent in his scientific architecture, exhibit commonalities with the grammatical approach. Here, the paper will tackle the issue of the science of urban design on two fronts; first, the relation between recursivity of patterns and cultural continuity; secondly, scientific analysis of history through form. 
First of all, the concept of recursivity emerges as a constant principle of ever-lasting change. Relations between successive periods in time enable to study change. Assumption of some continuity of forms through time, against which change can be measured, is fundamental. This type of continuity usually results from constraints placed on designer by cultural tradition which provide a repertory of forms and conventions. Formal grammars explore these forms and conventions and help to interpret the dynamic relation between them through a procedure of grammatical inference. This procedure consists of the subsequent phases of; abstraction of form from content, examination of plausible formal rules, derivation of evolution stages, and monitoring relationship of rules. Formal grammars analyse the possible connections between the ruptures in the formal structure and broken symmetries in the semiological reading of the city-text, thus, establishes parallel links with non-formal aspects of urban form and its evolution.

While Agrest (1991) describes city in terms of language, Barthes (1997), referring to Hugo, asserts that city is a text. One, then, is confronted, here, with the problem of intermediation between text and space. While Lefebvre defines space as a social practice, March (1998) suggests arithmetic and geometry, and Duncan (1990) suggests synechdoches and references for intermediating role (to encode and decode) between city-text and urban-space. Additionally, one may suggest formal grammars of diagrammatic and geometric relationships for analysing how culturally transmitted information may have been encoded in urban space (Çetin, 1999a).

At this point, it is of primary importance to encounter and emphasise a crucially significant argument presented in this paper. 'Rhythmic articulation ', in many types of transformation, remain constant whereas formal attributions always change. Therefore, the concept of rhythm as an underlying regularity forms the key element whereby it is a kind of principle recursivity which allows any kind of alteration or synthesis. Similarly, when architecture is considered as a language, grammars appear to be the underlying regularity which enable and regulate various kinds of interventions, alterations, additions and syntheses. When the historical examples of transformation are examined, it seems that those unchanging laws and underlying principles, independent from time and location, concentrate on either shapes or their spatial relationships. By the same token, they should be the features to refer in any form of intervention to urban scene rather than specific visual images. Therefore, shapes and spatial relationships compose that kind of grammar in urban context. Grammatical regularity offers us additional or alternative way of reading the essence of continuity in 'physical transformation through time'. This regularity appears to be deformed in times of radical changes in social, political, economical and cultural contexts within a particular location. One can presume city as a code whereby signs of urban disintegration could be identified. Note, here, the use of language not only as a metaphor but also as an analytical instrument, as Barthes (1997) once suggested.

Secondly, 'history of urban form' constitutes the core of 'scientific analysis in urban design'. The common points between science and history are their cumulative nature, as Rapoport (1990) points out, as well as their speculative nature. Their cumulative nature demands scrutiny of the object (i.e., urban form as a historic entity and scientific outcome) retrospectively. It also legitimises petrifaction of the object at any point of its evolution for analytical and descriptive purposes. The reading of urban form as an accumulation of transformation layers, places each intervention into the web of continuity which can be characterised by the multiplicity of readings. On the other hand, as one can 
find in Panofsky's idea of return to 'primitive modes of representation', speculations about past seem to have been a major source of creation throughout history of art. Because, structural reading of the past architecture determines the interpretation and, thus, demonstrates the importance of precedent in architecture. On the other hand, we are faced, along with the concept of speculation, with the crucial problem of interpretation (grammatical inference). Briefly, science of urban design is interested with the concept of recursivity in formal relations and with retrospective speculations in the evolution of formal relationships towards interpreting the connections between cultural aspects of urban space and formal language of urban morphology.

In addition to Fleshier's (1992) scepticism about the grammaticality of every form, Rowe's (1996) various interpretations of the facade of San Lorenzo clearly demonstrate how variable a formal analysis could be. Such a wide range in the flexibility of interpretation seems to constitute one of the major obstacles in describing urban space and its transformation in terms of formal grammars. This, however, should be clarified for the reader after the explanation of the procedure of grammatical inference. Moreover, the suggested idea of the link between grammatical inference and other constituents of design (such as designer, site forces, design process, social influences, chronology of events and design operations, symbolic content, etc.) may play important roles in overcoming of the problematic of interpretation.

Friedman's (1975) definition of history as a sequence of events in chronological order also raises the issue of whether this sequence is one of structured order or that of chance. In his argument, it is also suggested that histories of different parameters dependent on the same chronology could be correlated. Thus, scientific analysis of history brings about the idea of historically informed analysis which finds its manifestation in chronologically informed grammar analysis. The only apparent conflict between science and history remains to be the difference between them with regard to the degree to which their natures are analytical. As a result of this argument, one might suggest that, in order for 'urban design' to be spoken properly, study of the history of urban form has to be consolidated with the analytical component. In that sense, grammars promise to provide this analytical insight into the study of urban-architectural history.

Therefore, the science of urban design advocated here is theoretically grounded on the following principles.

Firstly, the concept of continuity is defined as the reproduction of hitherto accepted pattern.

Also, architectural tradition of a community is defined as a set of normative rules that can lead vernacular building process to reproduce a known and socially accepted pattern.

Thus, buildings can be assessed in terms of transmission of culture by artefacts. Architecture and urban design involve both culturally bound and universalistic components. The latter is based on comparative knowledge of forms and functions and aimed at innovation rather than cultural reduplication inherent in the former. Such abstract comparative thought suggests exploitation of encoded patterns. Urban space, in which layers of transformation is juxtaposed, is considered as a primary means by which urban transformation as cultural transmission is conceived by the inhabitants of this space. In that sense, this study concentrates specifically on urban space to decode the constant formal principles of this cultural transmission. 


\section{Grammars as the basis of cross-cultural studies}

Do we speak the language of 'urban design' properly? Do we grasp its grammar entirely? At this stage, one needs to briefly gather above discussions and arguments towards establishing a linguistic method to be able to conduct cross-cultural studies. Within the scientific framework of urban design, the formal language of urban morphology should be investigated, here, so as to introduce an analytical dimension into the study of urban-architectural history. Above-defined genetic logic manifests itself in various patterns because they are, basically, rule-based systems that regulate both the city form as well as the cyclic, recursive and rhythmic nature of urban activities. One cannot fail to admit that formal analysis (of such patterns) is essential, as mentioned before, both for composing a new city-text and reading (and interpreting) existing city-text (which forms a prerequisite for the former). Moreover, formal analysis acts as a syntactic way of studying of urban form to arrive at a semantic interpretation through scrutiny of the fragments of city-text. Therefore, what is called 'deep structures' of urban space should be examined through its morphological aspects in order to define a context in evolution (Conzen, 1978). Thus, grammars appear to be a potential common ground for assessing interventions into such historical contexts as well as a probable indicator for semiological disintegration by means of grammatical ruptures. Along this route, recursivity of formal patterns can be associated with cultural ones while scientific analysis of history can be achieved through investigation of formal evolution.

Furthermore, both science and art accommodate intermediate levels of complexity in terms of their own patterns. Thus, pattern structures also represent the reconciliation of science and art, and further demonstrate shared language and common values of urbanism through which culture-specific values are conveyed. Grammars (as a code or function of patterns) offer a useful device for intermediation between social and geometric spaces in decodification of fragments of city-text.

Therefore, urban transformation is analysed, here, in terms of rule-based, compositional systems called 'formal grammars'. It is observed that there is a linguistic logic (in design of city-form) which is composed of an initial form of the design, a set of possible rules applied to this initial form, and recursive structures which define the sequence, order and location of the rules applied to the initial form. It is suggested that recursive structures seem to remain the same within a particular design approach, a designer, a period, a society, etc. although forms and rules might change. This logic which is derived from shape grammars can be adapted to urban context. In order to adopt the methodology into the field of urban renewal, it is necessary to simplify the complex structure of urban transformation by classifying this structure into its components and by analysing them separately. Proposed model brings the issues of form and transformation of urban space into light at three interconnected levels in order to unfold the components of urban space: spatial configuration, boundary conditions, and regulating elements. The first level elucidates figure-ground relationships, and is determined either by peripheral rules or field formations; the second level delineates facade characteristics, and finally, the last level of grammatical transformation explicates axes, grids, layers and fields. The relations among these levels of transformation can reveal grammatical characteristics of a space. Thus, formal grammars may become a rule of thumb for communication in 'urban design' language. 
It is of prime importance to analyse the actual urban spaces and their compositional characteristics via suggested methodology. Whether morphological characteristics are a manifestation of a set of geometric rules applied to the form of space, and whether the transformation of specific spaces are expression of the recursivities of these set of rules in relation to the socio-cultural events that have shaped the space, are of particular interest in formal grammar analyses. The suggested methodology should be able to clarify and classify these rules so as to explain the evolution of space in terms of both form and content. Therefore, a comparative case study is conducted to observe the differentiations of formal vocabulary and grammar in different contexts.

\subsection{A case study: comperative analysis of urban contexts in Italy and Turkey}

As discussed above, the suggested methodology (formal grammar analysis) for analysing urban spaces, their morphology and transformation on a scientific basis (based on architectural form and its relation to architectural history, theory and sociology) is defined as a rule-based system and is inspired from the architecture - language analogy. Here, the methodology is applied to a comperative case in order to be able to reveal how the formal language (i.e., vocabulary, syntax and grammar) vary in differing contexts.

Figure 1 Superimposition of shared underlying compositional systems both in San Marco and Taksim Squares

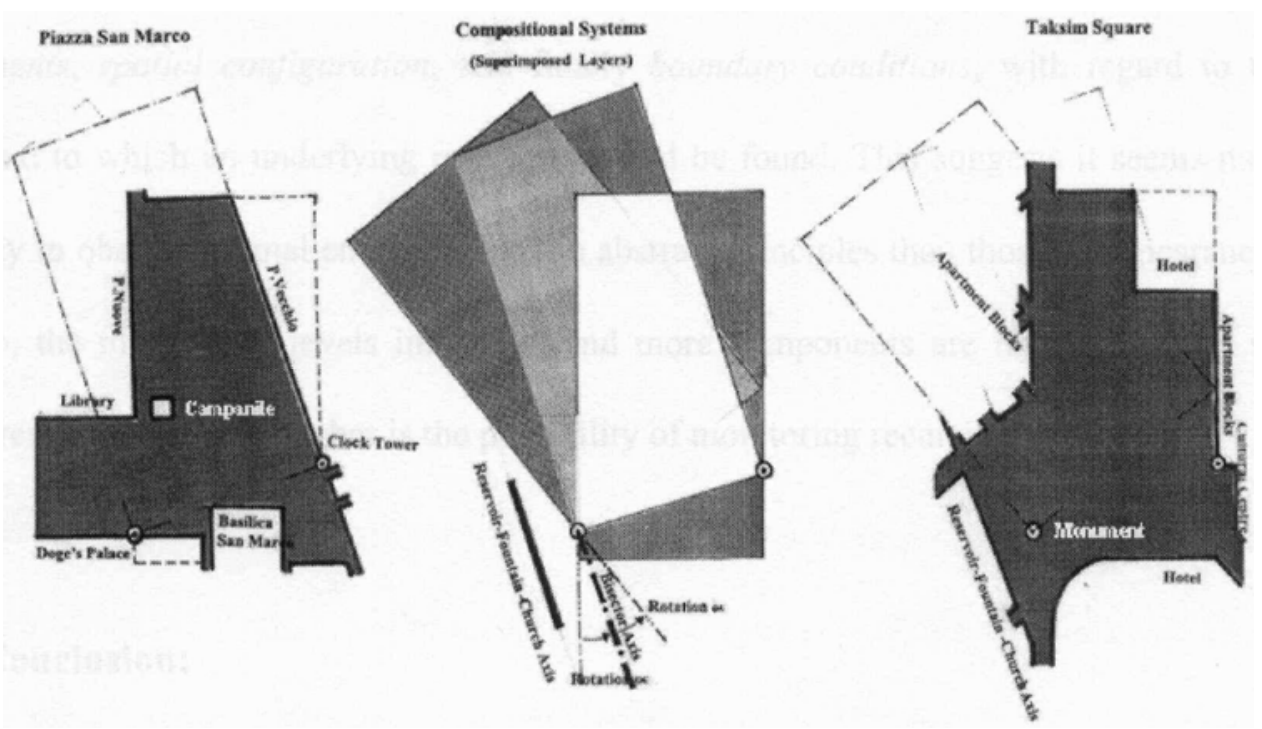

For instance, in a formal grammar study where Piazza San Marco and Taksim Square are analysed (Çetin, 1999b), both squares seem to be formed by superimposition of two organising layers (GRIDS) rotated around a pivotal point (Figure 1). Although one of those rotation points takes place on the periphery of the square and the other one is located within the square, both are articulated as landmarks (i.e., bell-tower and clock-tower). Chronologically, former case displays a very consistent relationship among the geometric rules that shape both layers. However, the relationships between the two organising layers in the latter seem to have been formed, via a very chaotic process 
resulting in an irregular pattern of geometric rules through successive stages of spatial transformation (Figure 2). Spatial forms (e.g., square, triangle and circle) can be considered as added, subtracted, divided, distorted, erased, scaled and rotated throughout the formation process of squares. Organising features (such as axes, grids) appear to regulate the formation of both spaces and masses in relation to each other. The analyses show that rules of spatial formation do not, even in San Marco Square, narrate a logical sequence by themselves. These rules can demonstrate an ordered structure only when considered in combination with the rules of organising features.

Figure 2 (a) Evolution of Piazza San Marco when analysed in terms of rule-based system of formal grammars (b) evolution of Taksim Square

PIAZZA SAN MARCD [initial form]

hYPOTHETICAL RECONGTHUiction

GRAMMATICAL INFERENCE
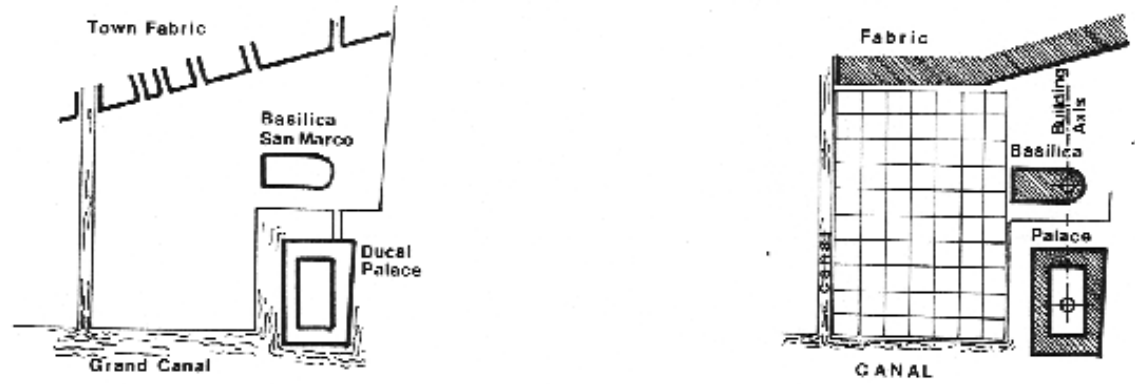

$900 s$

pIAZZA BAN MARCo (phase i)

hYPOTHETICAL. hECONBTHUCTION

GRAMMATICAL INFERENCE
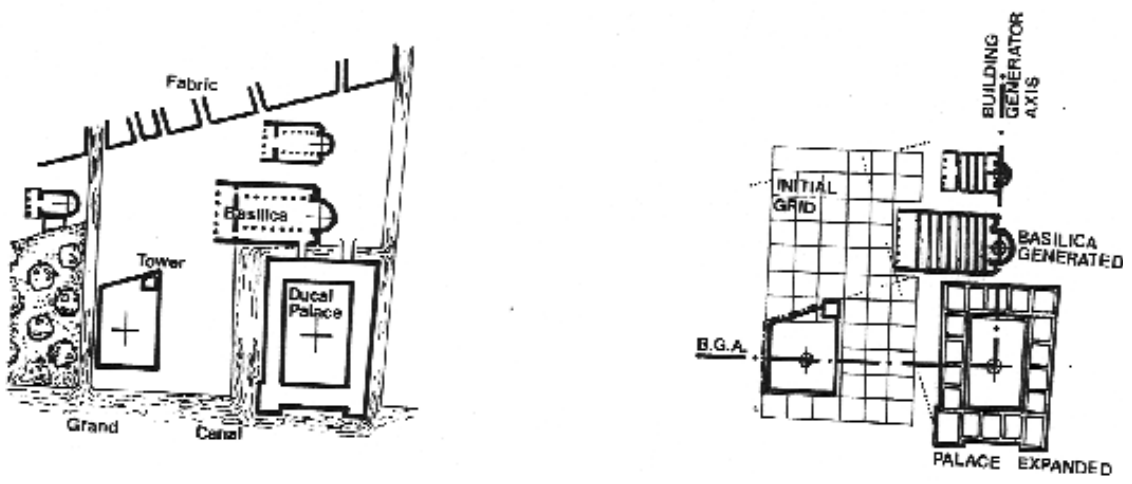

11008 
Figure 2 (a) Evolution of Piazza San Marco when analysed in terms of rule-based system of formal grammars (b) evolution of Taksim Square (continued)

piazza gaN MARco (phage II)

HYPOIHEIICAL RECONSTRUCTION

GRAMMATICAL IMFERENCE
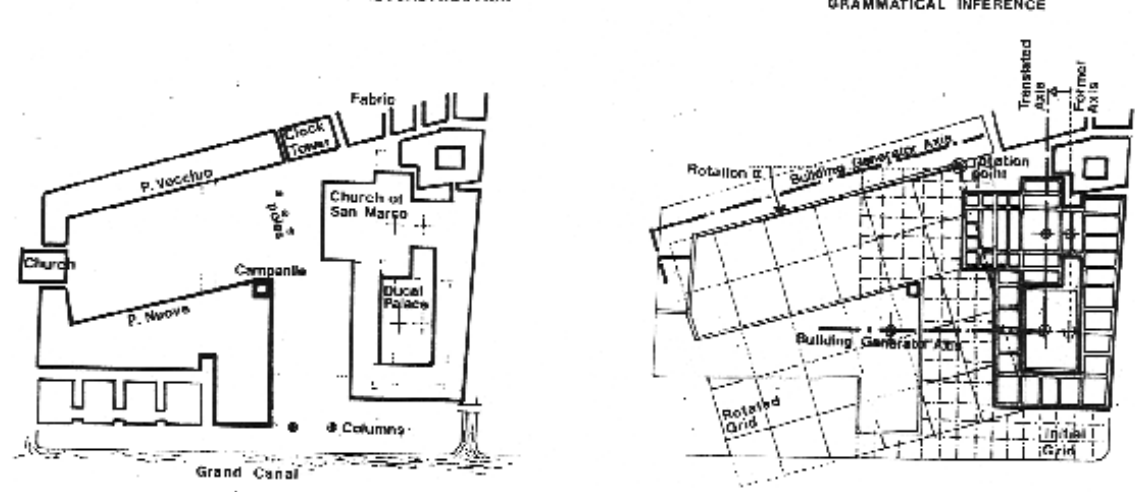

1300 e

PIAzzA BAN MARCo (phage III

MYPOTHETICAL RECDHBTRDCTION

GRMUMATICAL INFERENCE
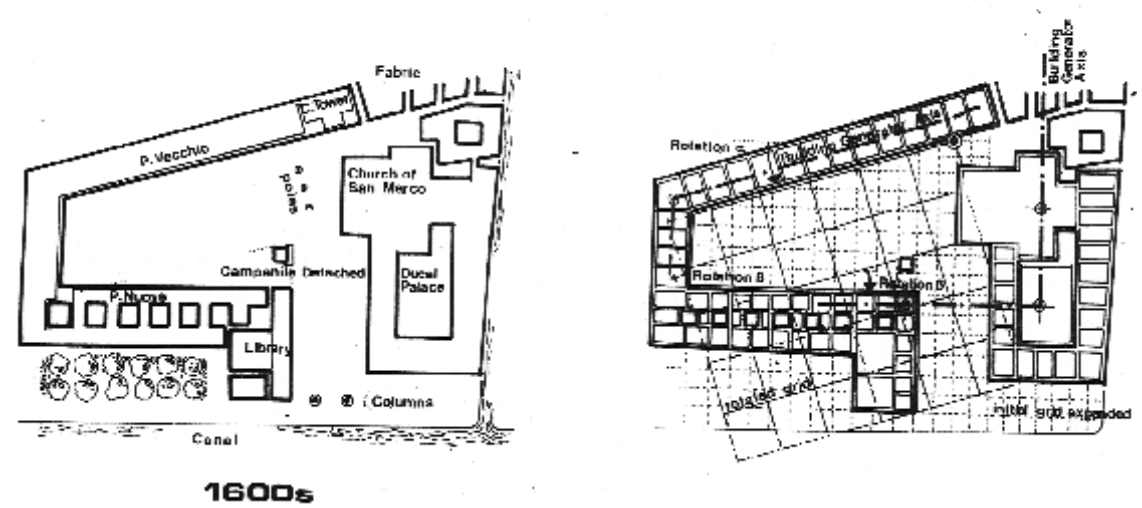

(a)
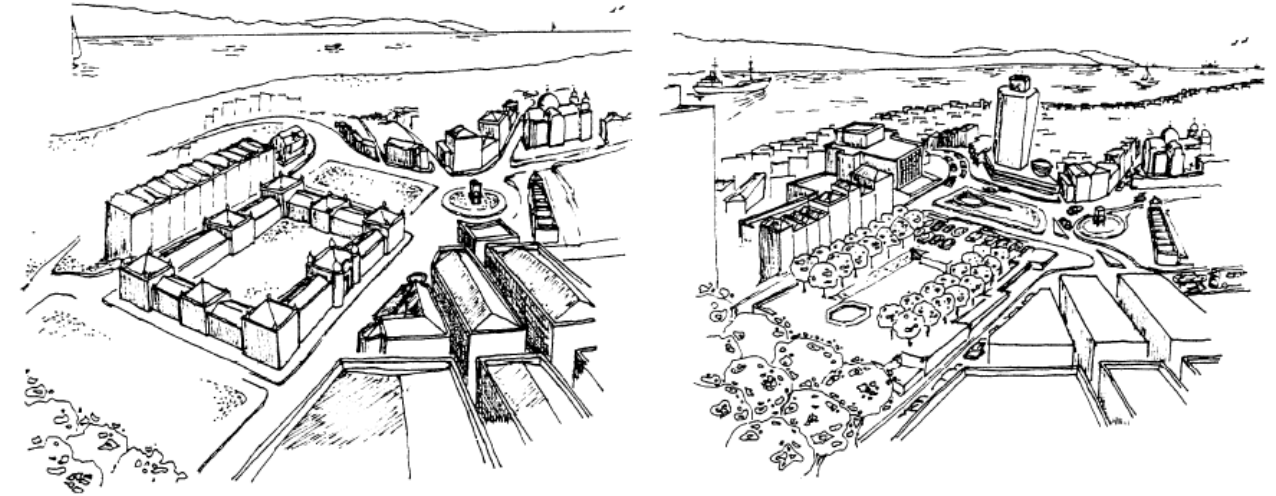

(b) 


\subsection{Formal analysis and empirical data}

Each organising layer seems to embody their own rules that shape spaces associated with these layers (Figure 3). One can deduce from these cases that the relationship between the rules of both layers seems to determine the grammatical character of each space. The range of selection from a wide spectrum of rules in one square seems to differ profoundly from the other although basic rules (such as addition, subtraction, and rotation) are shared in both cases (Figure 4). While San Marco Square is dominated by rules associated with its periphery (to define modifications in spatial configuration), Taksim Square is characterised by field formation rules and division rules (due to dense vehicular traffic) (Figure 5). Also, the relation between the axes and building generator points exhibit a peripheral nature for the defining the spatial modifications in the former case, whereas such an axial tendency is not clear enough to define a peripheral character in the latter (Figure 6). In San Marco Square, these rules seem to display an order whereby a certain sequence of rules - working for one layer - is alternated by another rule - serving for the formation of the other rotated layer (in chronological order). Taksim Square, however, shows (at certain phases of transformation, particularly in 1960 and 1980) an evident concentration of rules on the formation of only one layer, resulting in an irregular grammatical order in its relatively short lifetime. When the sociological reasons behind such a morphological process are historically investigated, one could notice the fact that social, cultural, economic (in)consistencies in the production process of space are strongly connected to its geometric properties. In other words, as the social events that shape physical spaces change, the intrinsic deep-structures that regulate their formation also varies. Doubtlessly, similar forms, that is to say, similar vocabulary, can be used for various spaces in different times and contexts. However, the grammars profoundly differ, which makes urban-architectural language quite specific to its context. Thus, one can suggest that formal grammars are contextual and space-specific. As can be seen, these grammatical structures (comprised of various morphological) components convey different messages for each square, and the combinations of their components display different patterns, and thus, exhibit dissimilar grammatical characteristics. Hence, these combinations may constitute a non-verbal language for communication among different parties shaping urban spaces, and among successive generations of designers working on the same context. To say more clearly, urban-architectural intervention can be considered as a matter of re-reading the ongoing language, and re-writing the existing urban texts in the city-escape.

As a matter of fact, the main argument is, not to reveal those common points only (neither is to merely reveal dissimilarities) in two cases, but to point out the observation that as further components are introduced to grammatical inference in the formal analysis process, it becomes more likely to find underlying grammatical patterns within the stages of transformation, and particularly within the range defined between extreme predictability and extreme entropy. This means, regularities (recursive structures) are expected to be neither too clear nor too complex so as to be capable of expressing an underlying logic. The results of analyses also indicate the possibility that these grammatical patterns display a strong association with social structure in which the urban space is shaped. Therefore, it would be appropriate to suggest that formal grammars are capable of inferring grammatical structure only within a clearly defined range of patterns. Curiously, the analysis of the cases indicates that three major levels of grammar analysis exhibit a descending order starting with regulating elements, spatial configuration, and 
finally, boundary conditions, with regard to the extend to which an underlying regularity could be found. This ranking suggests that it seems more likely to observe formal encodings within abstract principles than those in appearances. Also, the more these levels are integrated and additional components are introduced into the inference process, the higher is the possibility of monitoring recursivities. These recursivities (observed in abstract principles), may encode rudiments of continuity in a language. In Venice, architects seem to have managed speaking a common urban design language throughout the successive ages. In Taksim, however, there seems to be a chronic miscommunication issue due to irregularities of grammatical structure in the language of urban space design. Consequently, all types of language (spoken, written, formal, architectonic or stylistic) are the ramifications of a specific culture and seem to be in a coherent interaction with each other.

Figure 3 Graphic representation of the superimposition of regulating fields and its relation to rule-based system of building generation in Piazza San Marco
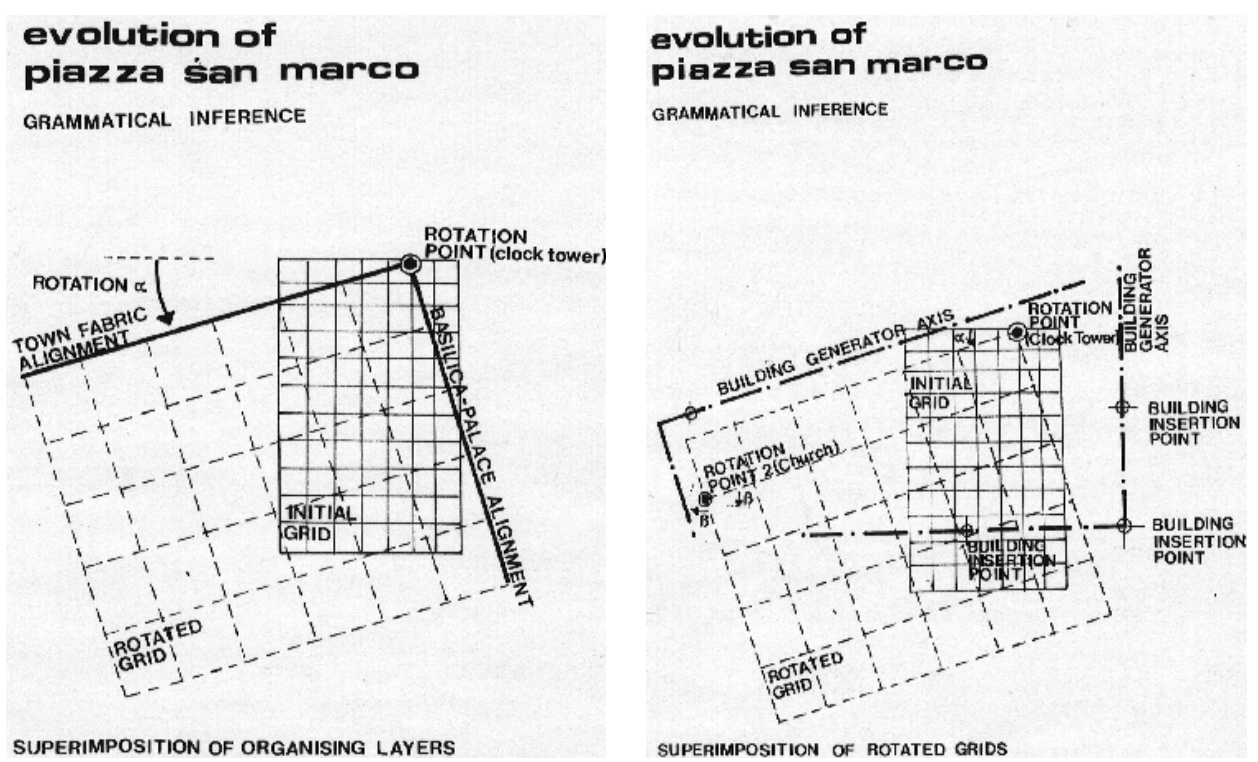

However, when applied to empirical analyses of actual cases, formal grammars encounter certain limitations. In this study, for instance, one must stress that a chronological comparison is never intended because Piazza San Marco finished its actual transformation approximately when Taksim Square had started to develop as an urban space. Formal features constituted the basis and scope of the study. Furthermore, the differences between the socio-political and cultural contexts of both squares should also shed light into the formal comparisons. Overall, one can suggest that consistency of morphological patterns implies the existence of a formal language in urban-architectural context, and reveals recursive character of its socio-political context. Thus, one would be able to interpret architectural tradition and cultural context by taking advantage of formal grammars, as one utilises the language to access the essentials of a culture. Besides, such a methodology would enable the interventions to speak properly in a specific cultural context. 
Figure 4 The underlying system of building generation rules valid in Piazza San Marco

\section{RULES}

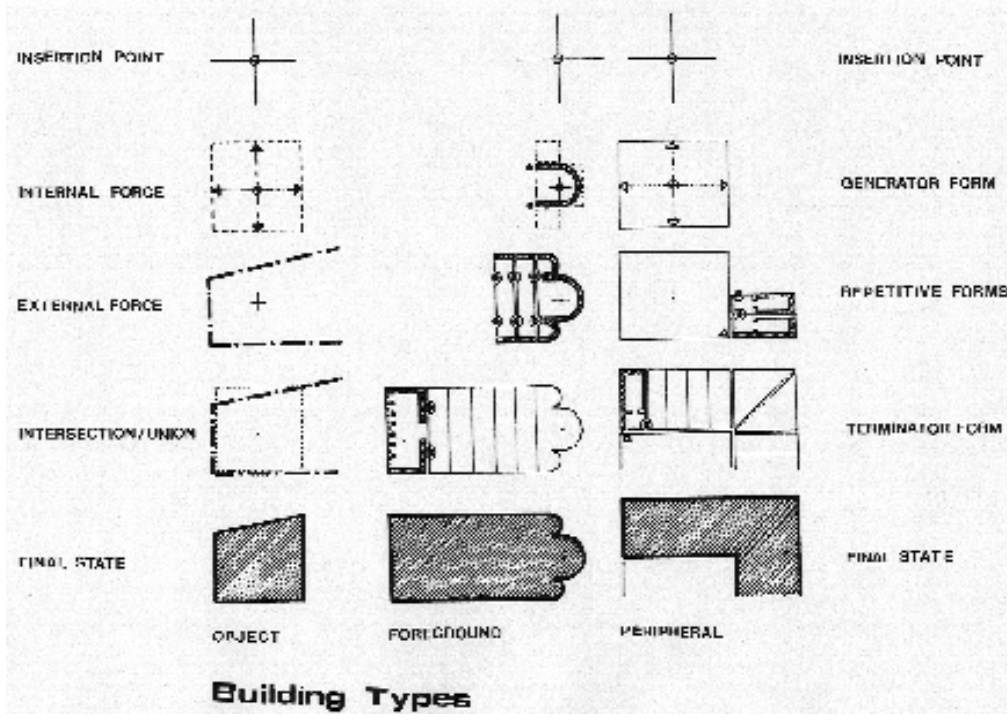

Figure 5 Field rotation rules and division rules associated with dense traffic layout in Taksim Square

Taksim Square

GRAMMATICAL INFERENCE

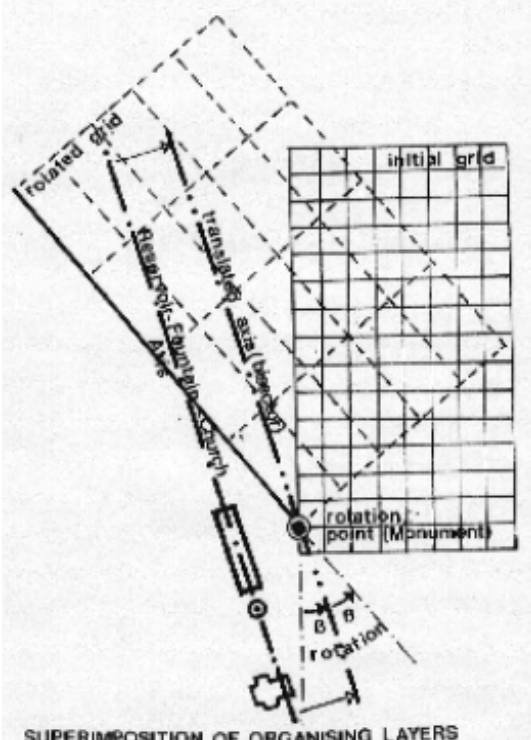

\section{taksim square}

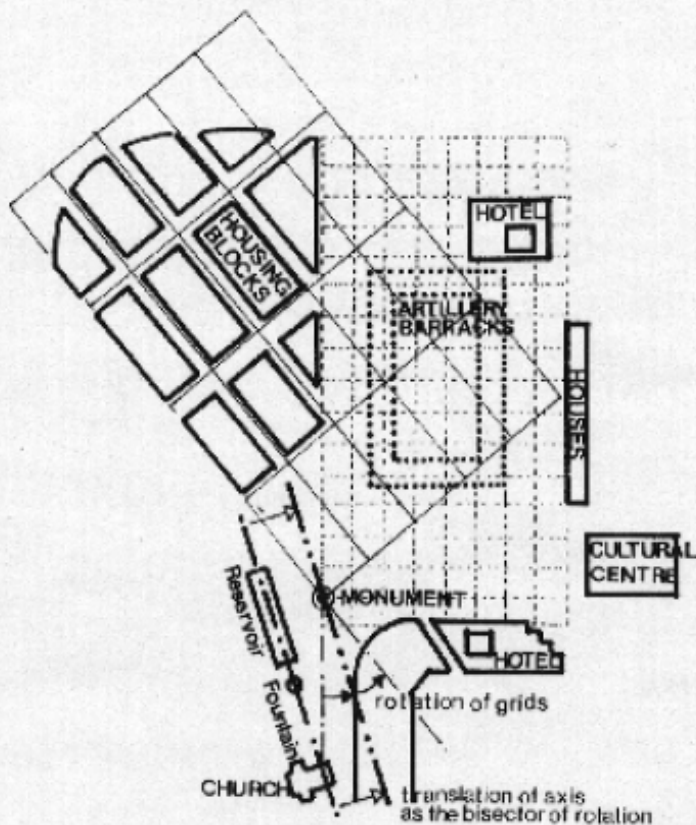


Figure 6 Building generator rules exhibiting different character regarding their peripheral nature in two different squares
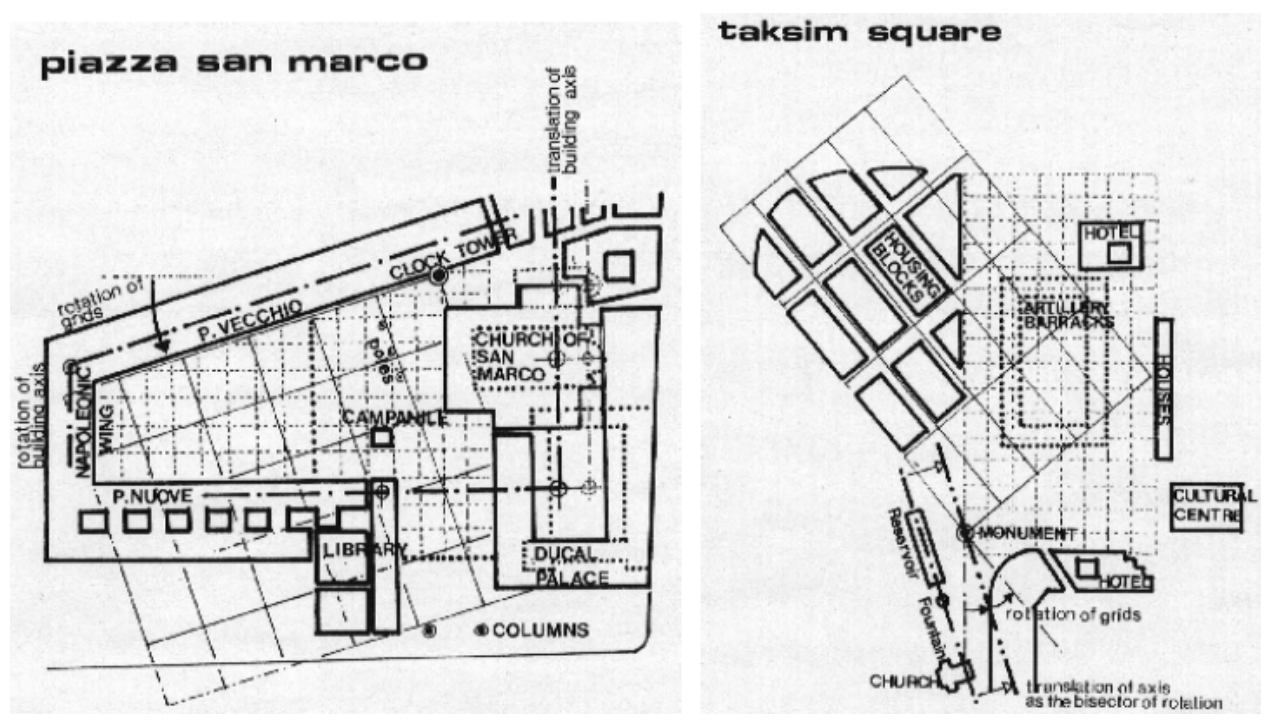

\subsection{Discussion}

The paper discusses that various aspects of urban contexts could be unveiled through formal abstraction on a systematic basis. One must note that Alexander (1965) mathematically explains the richness and complexity of traditional city by some sort of formal language (semilattice). Hillier (1993) attempts not only to relate 'human interaction' to the 'geometry of urban structure', but also to associate the 'human movement' with a 'spatial syntax'. Also, Salingaros (2001) accentuates the link between 'life' and 'urban geometry'. He asserts that the mess of geometrical configurations in traditional town fabrics appears to support multiplicity of life through spontaneous interaction. He conceives a 'fine grained geometry that allows mixing' as a 'prerequisite for life'. He even associates geometry with ecology in the sense that both are complex overlapping systems composed of organisms of different sizes. Furthermore, the evolution of urban morphology as the changes of the connections in a geometrical configurations in a town fabric over a range of time.

Urban design is conceived as an instrument to provide formal continuity of urban transformation by addressing the deep structures of urban context. The roots of this understanding lie within the compositeness of context and its recursive and pattern-like nature. In that sense, their rule-based common characteristics are suggested as the platform of reconciliation to overcome the lack of analytical capacity, and it is suggested that city (when described as text) might have an underlying grammar and syntax. Thus, the relation between the cyclical nature of the activities within city, and the recursive and pattern-like quality of physical urban form is investigated in terms of deep structures in town fabric and those in its transformation. The patterns, corresponding to 
specific morphological factors, are considered as agents that synthesise dualities into single form.

Another important issue is that meaning in urban environment lies in the translation of formal code (if city is text and architecture is its language) into non-formal one, and the following argument is that if a formal code has recursive and pattern qualities, its translation, thus, meaning may have similar qualities. This may form the basis of links between the intrinsic formal logic and non-formal aspects of urban transformation. Eco (1997) also supports the idea of a separate formal code in urban environment and appropriateness of its study in isolation from other codes. Thus, urban transformation as a form of encoded cultural transmission, and environmental meaning as a form of non-verbal communication both share a common underlying code, as Eco suggests. Therefore, the intended argument of this study is that form and its recursive and grammatical characteristics may constitute Eco's concept of underlying code, and thus, provide a methodological insight into the relation between text and space in urban context.

Actually, even a brief glimpse at the evolution of art and design demonstrates subsequent critical movements each distinguished from the preceding period by the opposite design theory on which it is based. While one of these two opposing theories emphasises the importance of the designer (artistic subjectivity), the other stresses the external conditions and determinants (rational objectivity) on design form. Strange as this may sound, many theories discuss form as a product of either one or the other view, whereas one who examines history of design and architecture cannot fail to notice that these two views complete each other like Gestalt diagrams. Recent syntactical (Hillier and Hanson, 1984) and grammatical theories (Stiny and Gips, 1975) tend to incorporate a synthesis of designer's mind and external conditions as determinants of form (Gelernter, 1995). They appear to have developed these structuring activities, their evolution in time, into 'deep structures' of the spatial organisation of buildings or cities, on the basis of design knowledge organised in terms of rules and types (Schon, 1988). Therefore, it would be appropriate to assert that linguistic approaches to the study of urban space may help to explicate urban issues associated with form in a systematic manner as the evidence above would support the argument.

\section{Conclusions}

In this study, it is intended to consider urban-architectural transformation as a language, and its underlying grammatical recursivity as the intermediating agent between the fragments of the city-text and new spatial essays inserted into it. Formal grammars are utilised as an additional methodology to reveal latent regularities in the transformation of urban spaces. The two cases selected from Western and Eastern urban spaces are scrutinised via this methodology. It is observed that both exhibit morphological similarities and organisational differences consistent with their respective socio-political stability. In other words, the differences in socio-economic, political and cultural conditions encode different formal grammars in different contexts. Urban-architectural science intends to reveal these formations by deciphering the 
morphological structure of townscape into rule-based geometrical systems, and by chronologically associating these analyses with the conventions of architectural history, theory, and sociology.

Obviously, this formal grammar approach is not meant to replace other scientific approaches of urban-architectural history and urban morphology, rather add to it and complete with the missing bulk of syntactical information possibly inherent in urban environment. Yet again, it is believed that similar conceptual studies would pave the way leading to a historico-geometrical understanding (in addition to historico-geographical approach of Conzenian School) of urban research, and would witness a growing awareness of grammatical and syntactical analyses. Consequently, one can not fail to notice that the overlap between urban morphology and shape grammars promises a hybrid rigour.

Although, throughout the paper, urban design seems to be treated primarily as a matter of formal composition, formal attributes are taken as a tool and finally, discussion is also extended to how it can be linked to other aspects of its content. In contrast to Vesely's (1985) confirmation of Perez-Gomez in that symbolic representation is being transformed into instrumental representation since the 18th century, in today's context of multi-disciplinary context of science, a semi-autonomous and self-referential representation based on formal attributes can only be intended as instruments by which parallel links can be established with other aspects of the phenomena. Because when liberated from its dogmatic ties, geometry would offer us again the benefit of unfolding even further horizons towards describing many complex and irregular phenomena in natural and manmade world. Therefore, whether a plausibly underlying code in the city form (and its transformation) may have parallelism with the non-formal aspects of the city (such, as social, economic, politic, symbolic, functional, etc.) should be the main concern for further grammatical studies. Because, in the light of the cases examined above, urban-architecture seems to utilise the grammatical characteristics of a universal language in order to convey the assets of local culture. The formal vocabulary can be confronted in any part of the world, however, the configuration and its underlying grammatical logic is extremely site, space and time specific. Although one might also speculate about the potential uses of formal grammar approach in urban analysis, in assessment of urban renewal proposals, in teaching of architectural history, in CAD and GIS, I must conclude that it should be only conceived as a useful instrument to extend the language of understanding of environment prior to its design. Particularly in an era when computational sciences, technology and design is rapidly heading towards a level of artificial intelligence, humane values regarding urban life should urgently be re-defined in a new format. Formal grammars seem to be promising for transferring traditional urban attributes into the next generation and into the new medium of information. In order for architects to be able to speak 'urban design' properly and to decode the fragments of city-text, it seems necessary to grasp spatial grammars well enough. 


\section{References}

Adams, W. (1999) 'Cosmological events as ordering determinants of the great houses of Chaco Canyon', Environments BYDESIGN, Vol. 2, No. 2, pp.153-175.

Agrest, D. (1991) Architecture from without: Theoretical Framings for a Critical Practice, MIT Press, Cambridge Mass.

Alexander, C. (1965) 'City is not a tree', Architectural Forum, Vol. 122, No. 1, pp.58-62.

Barthes, R. (1997) Leach, N. (Ed.): Semiology and the Urban, Rethinking Architecture: A Reader in Cultural Theory, Routledge, New York.

Çetin, M. (1999a) 'The grammar of urban transformation: a tale of two cities', ACSA International Conference Proceedings (La Cittâ Nuova - The New City), 29 May to 2 June, pp.417-422, ACSA Press, Rome; Washington DC.

Çetin, M. (1999b) 'Principles of urban transformation: a grammatical interpretation of two urban squares', Proceedings of ISUF'99: Sixth International Seminar on Urban Form (Transformations of Urban Form: From Interpretations to Methodologies in Practice), 23-26 July, Florence, Alinea Editrice, FM2.7-10.

Conzen, M.P. (1978) 'Analytical approaches to urban landscape', in Butzer, K.W. (Ed.): Dimensions of Human Geography: Essays in some Familiar \& Neglected Themes, Research Paper No. 186, Dept. of Geoography, University of Chicago.

Doxiadis, C.A. (1972) Architectural Space in Ancient Greece, MIT Press, Camb. Mass.

Duncan, J.S. (1990) The City as Text: The Politics of Landscape Interpretation in the Kandyan Kingdom, Blackwell, London.

Eco, U. (1997) 'Function and sign: the semiotics of architecture', Leach, N. (Ed.): Rethinking Architecture: A Reader in Cultural Theory, Routledge, New York.

Fleshier, A. (1992) 'Grammatical architecture?', Environment and Planning B: Planning and Design, No. 19, pp.221-226.

Friedman, Y. (1975) Lang, C. (Ed.): Towards a Scientific Architecture, MIT Press, Camb. Mass.

Gelernter, M. (1995) Sources of Architectural Form: A Critical History of Western Design Theory, Manchester UP, Manchester.

Gosling, D. and Maitland, B. (1984) Concepts of Urban Design, Academy ed., London.

Guadet, J. (1894) Elements et theories de l'Architecture, Librarie de la Construction Moderne, Paris.

Herbert, T. and Sanders, I. (1994) 'African shape grammar: a language of linear Ndebele Homesteads', Environment and Planning B: Planning and Design, No. 21, pp.453-476.

Hillier, B. (1993) 'Specifically architectural theory: a partial account of the ascent from building as cultural transmission to architecture as theoretical concretion', The Harvard Architecture Review, No. 9, pp.9-27.

Hillier, B. and Hanson, J. (1984) The Social Logic of Space, Cambridge UP, Cambridge.

March, L. (1998) Architectonics of Humanism, Academy ed., London.

Rapoport, A. (1990) History and Precedent in Environmental Design, Plenum Press, London.

Rowe, C. (1996) 'Transparency: literal and phenomenal', Caragonne, A. (Ed.): As I Was Saying: Recollections and Miscellaneous Essays, MIT Press, London.

Rykwert, J. (1976) The Idea of a Town, Faber \& Faber, London.

Salingaros, N.A. (2001) 'Remarks on a city's composition', RUDI - Resource for Urban Design Information. 
Schon, D. (1988) 'Designing: rules, types and worlds', Design Studies, Vol. 9, No. 3, pp.181-190.

Simmel, G. (\{1908\}1955) Wolff, K.H. and Bendix, R. (Eds.): Conflict and the Web of Group Affiliations, Illinois Press.

Stiny, G. and Gips, J. (1975) Pictorial and Formal Aspects of Shape and Shape Grammars, Birkhauser, Brussels.

Thiis-Evansen, T. (1988) Archetype in Architecture, Norvegian University Press, Oslo.

Vesely, D. (1985) 'Architecture and the conflict of representation', AA Files, No. 8, pp.21-38.

Whitehand, J.R. (1987) Changing Face of Cities: A Study of Development Cycles and Urban Form, Basil Blackwell, Oxford.

Wightman, G. (1997) ‘The Imperial Fora of Rome’, ISAH, Vol. 56, No. 1, pp.64-85. 